\title{
Evaluation of biomarkers for cardiotoxicity of anthracyclin-based chemotherapy
}

\author{
F. J. F. Broeyer · S. Osanto $\cdot$ H. J. Ritsema van Eck $\cdot$ \\ A. Q. M. J. van Steijn · B. E. P. B. Ballieux • \\ R. C. Schoemaker · A. F. Cohen $\cdot$ J. Burggraaf
}

Received: 18 September 2007 / Accepted: 28 February 2008 / Published online: 15 March 2008

(C) The Author(s) 2008

\begin{abstract}
Introduction The clinical assessment of the myocardial damage caused by anthracyclin (ANT)-therapy is difficult. Therefore a study was performed to evaluate non-invasive markers of anthracyclin-induced cardiac effects, with emphasis on course-to-course variation.

Methods Eligible for study participation were patients, without known cardiologic abnormalities who did not use cardiotoxic medication (except for ANT-therapy), who had previously completed at least three cycles of anthracyclincontaining chemotherapy $(n=14)$ and patients who were ANT-naïve and who were scheduled to receive doxorubicin-containing chemotherapy $(n=12)$. Seven patients in this last group also completed at least three cycles and were available for follow-up assessments; thus a total population of 21 patients $(12 \mathrm{~F} / 9 \mathrm{M})$ completed at least three courses ANT-chemotherapy. In these patients blood samples and ECG-recordings were taken within 6 months after completion of ANT-therapy. In 12 patients $(10 \mathrm{~F} / 2 \mathrm{M})$ assessments were also done before, immediately afterwards and at $24 \mathrm{~h}$ after each course of ANT.

Results and Conclusions In the patients who completed chemotherapy, NT-proBNP was $277 \%(n=21 ; 95 \%$ CI: $86-661 \%, P<0.001)$ higher compared to healthy volun-
\end{abstract}

F. J. F. Broeyer $(\bowtie) \cdot$ R. C. Schoemaker · A. F. Cohen .

J. Burggraaf

Centre for Human Drug Research, Zernikedreef 10,

2333 CL Leiden, The Netherlands

e-mail: fbroeyer@chdr.nl

S. Osanto · A. Q. M. J. van Steijn - B. E. P. B. Ballieux

Leiden University Medical Center, Leiden, The Netherlands

H. J. Ritsema van Eck

Advanced Medical Systems, Maasdam, The Netherlands teers. During the first course NT-proBNP rose $269 \%$ $(n=12 ; 167-409 \%, P<0.0001)$ at $24 \mathrm{~h}$ post-administration. The linear corrected QT (QTcL) directly after the first administration of ANT increased by $9.56 \mathrm{~ms}(n=12 ; 3.85$ $15.27, P<0.001)$ and this prolongation was still present at $24 \mathrm{~h}, 11.48 \mathrm{~ms}(n=12 ; 5.61-17.34, P<0.0001)$. Both NTproBNP and QTcL returned to baseline before the start of the next course and a similar pattern was observed during each course. NT-proBNP and QTcL may be useful markers for course-to-course evaluation of anthracyclin-induced cardiotoxicity.

Keywords Anthracyclines - Cardiotoxicity - Biomarkers · Electrocardiography

\section{Introduction}

Anthracyclines, such as doxorubicin (DXR), cause serious cardiac side-effects (Singal et al. 1997). Acute tachyarrhythmias and acute heart failure may occur after high doses, but these reactions are now rare due to changed dosage-schemes (e.g. slower infusion) with the aim to prevent this. However, the sub-acute or chronic cardiac effects of anthracyclines remain a clinical problem. Clinically, anthracyclin-induced cardiotoxicity manifests itself as left ventricular failure which develops insidiously over months to years after completion of the anthracyclin-based chemotherapy and may result in congestive heart failure (CHF) (Shan et al. 1996; Minotti et al. 2004).

Recent studies suggest an incidence of this type of cardiotoxicity of $5 \%$ in doses up to $400 \mathrm{mg} / \mathrm{m}^{2}$ increasing to $48 \%$ in subjects receiving $700 \mathrm{mg} / \mathrm{m}^{2}$ (Swain et al. 2003). But even at doses up to $150 \mathrm{mg} / \mathrm{m}^{2}$ CHF was occasionally reported (Swain et al. 2003). In addition to the cumulative 
dose, age, gender and dosing schedule have been reported as independent risk factors (Von Hoff et al. 1979).

The mechanism of anthracyclin-induced cardiotoxicity is not totally unravelled. It is likely that the decline in myocardial function is related to apoptosis of cardiac myocytes that occurs apparently at random in the myocardium (Thorburn and Frankel 2006). Anthracyclin-induced formation of reactive oxygen species (ROS) in the presence of intracellular iron, impaired homeostasis of intracellular iron and calcium (that may facilitate the apoptosis induced by the ROS) have been put forward as mechanisms. However, other possible mechanisms have been suggested and it is likely that anthracyclin-induced cardiotoxicity develops as a result of a large number of different insults (Minotti et al. 2004).

It is generally acknowledged that anthracyclin-induced cardiotoxicity becomes evident after completion of the chemotherapy. The gold standards to detect anthracyclininduced cardiotoxicity are cardiac imaging techniques or myocardial biopsy. However, these methods have either the disadvantage that cardiotoxicity is detected late, namely when decline in left ventricular ejection fraction (LVEF) already has occurred (imaging techniques) or that it is highly invasive and based on the assumption that the damage is equally distributed over the myocardium (biopsy).

Animal studies have shown that anthracyclin-induced apoptosis can occur already after a single dose (Bennink et al. 2004; Kumar et al. 2001; Arola et al. 2000). This is line with the finding in humans that even at low cumulative doses cardiotoxicity have been reported (Swain et al. 2003). If this could be measured and confirmed in humans, it might be possible to detect anthracyclin-induced cardiotoxicity in an early stage. Unfortunately, the assessment of apoptosis itself in humans is difficult, but it can be hypothesised that the cell loss in the heart may be detected indirectly. It is conceivable that cardiac damage results in leakage of cardiac enzymes into the circulation, conduction disturbances and that the loss of function will be compensated by autocrine cardiac mechanisms. Indeed, it has been reported that elevated concentrations of troponin, prolongation of QT-interval and increased levels of natriuretic peptides are associated with anthracyclin-induced cardiotoxicity after completion of chemotherapy (Meinardi et al. 2001; Daugaard et al. 2005; Kilickap et al. 2005; Cardinale et al. 2004). However, little information is available for the course-to-course effects of anthracyclines on these markers and thus they are not used to assess the early effects of anthracyclines on the heart. Additionally, the evaluation of interventions or treatments designed to prevent the damage requires a robust early and preferably quantitative marker of the damage. Hence, there is a need for biomarkers that can be used to detect early anthracyclininduced cardiotoxicity. Therefore a study was performed to evaluate non-invasive markers of anthracyclin-induced cardiotoxicity, with emphasis on course-to-course variation during four subsequent chemotherapy courses.

\section{Materials and methods}

Study population

The study was carried out in 26 patients with various malignancies who received DXR as chemotherapy, in combination with other chemotherapeutics (etoposide, vincristine, ifosfamide, actinomycine, docetaxel, cyclophosphamide, methotrexate, 5-flurouracil, cisplatin) and a group of healthy controls. One patient received epirubicin; for this patient the epirubicin dose was converted to the equivalent DXR-dose with the commonly used conversion factor of 0.5 (Launchbury and Habboubi 1993; Ewer and Benjamin 1996).

Eligible for study participation were patients who had previously (within 6 months) completed at least three cycles of anthracyclin-containing chemotherapy $(n=14)$ and patients who were anthracyclin-naïve and who were scheduled to receive DXR-containing chemotherapy $(n=12)$. Seven patients in this last group also completed at least three cycles and were available for follow-up assessments; thus a total population of 21 patients completed at least three courses ANT-chemotherapy. Of the anthracylcin-naive group the majority $(n=9)$ received DXR combined with cyclophosphamide for breast cancer. Two patients were treated with DXR and cisplatin for a carcinoma of the endometrium and osteosarcoma, respectively, and one patient received DXR in combination with vincristine, etoposide and ifosfamide for an Ewing sarcoma. The median number of courses of this subpopulation was 4 ; four patients received more courses, namely 5, 6 and 8 courses, respectively.

Patients and volunteers with known cardiac abnormalities, e.g. symptoms of angina pectoris, myocardial infarction or other myocardial abnormalities, or receiving other drugs with a known or suspected cardiotoxic potential and patients with clinically significant abnormalities, other than those related to their malignancy, were excluded form the study. Subjects did not use any QT-prolonging agents, except for 5-HT3-receptorantagonists for the prevention of nausea. Especially patients with an impaired renal function, haemoglobin level below $5 \mathrm{mmol} / \mathrm{L}$ or other clinically significant laboratory abnormalities other than those possibly related to their disease were excluded. A summary of the demographics of the patients and the volunteers is given in Table 1.

The Medical Ethics Committee of Leiden University Medical Center (LUMC) approved the protocol for this 
Table 1 Subject characteristics and cumulative doxorubicin dose

BMI, age and the estimated glomerular filtration rate (using the MDRD formula) are given as mean $\pm \mathrm{SD}$. The cumulative dose is given as median with the interquartile range

ANT anthracyclin, BMI body mass index

\begin{tabular}{|c|c|c|c|}
\hline & \multicolumn{2}{|l|}{ Patients } & \multirow{2}{*}{$\begin{array}{l}\text { Controls } \\
(n=14)\end{array}$} \\
\hline & $\begin{array}{l}\text { Completed }>3 \text { course } \\
\text { ANT-chemotherapy }(n=21)\end{array}$ & $\begin{array}{l}\text { ANT-naive } \\
(n=12)\end{array}$ & \\
\hline Age (years) & $46 \pm 15$ & $48 \pm 13$ & $46 \pm 14$ \\
\hline Gender (F/M) & $12 / 9$ & $10 / 2$ & $8 / 6$ \\
\hline $\mathrm{BMI}\left(\mathrm{kg} / \mathrm{m}^{2}\right)$ & $22.9 \pm 2.7$ & $25.3 \pm 2.1$ & $23.0 \pm 2.1$ \\
\hline Cumulative doxorubicin dose (mg) & $520(270)$ & $440(138)$ & NA \\
\hline $\begin{array}{l}\text { Estimated glomerular filtration } \\
\text { rate }(\mathrm{mL} / \mathrm{min})\end{array}$ & $84.5 \pm 26.4$ & $86.2 \pm 21.2$ & NA \\
\hline Haemoglobin $(\mu \mathrm{mol} / \mathrm{L})$ & $6.9 \pm 1.1$ & $7.8 \pm 0.8$ & NA \\
\hline
\end{tabular}

observational study. All patients were included after giving written informed consent to participate.

\section{Study outline}

For each patient assessment consisted of blood sampling and ECG-recording within 6 months after completion of the chemotherapy. In the subpopulation of anthracyclin-naïve patients $(n=12)$ measurements were made at each chemotherapy course. For comparability only data from the first four courses were used in the analysis. At each of these assessments blood sampling and ECG recording were done before $(t=0)$, at completion of the chemotherapy infusion $(t=4)$ and at $24 \mathrm{~h}(t=24)$ after the drug administration. For the controls a single assessment was done.

\section{Medication}

All chemotherapy was prepared by the pharmacy of LUMC according to the applicable guidelines. The median total volume load for the anthracyclin-naïve subpopulation during the courses was $250 \mathrm{~mL}$ over $2 \mathrm{~h}$.

Sample handling and assays

Blood samples were centrifuged immediately after collection and serum/plasma was stored at $-40^{\circ}$ until analysis. The samples were analysed for cardiac troponin $\mathrm{T}$ (cTnT), the mass concentration of creatine kinase-MB (CK-MB mass), atrial natriuretic peptide (ANP) and the N-terminal propeptide of B-type natriuretic peptide (NT-proBNP). Each individual assay was performed batchwise to avoid interassay and interindividual variability using automated and validated assays at the Central Laboratories for Clinical Chemistry of LUMC. The concentrations of cTnT and NTproBNP in serum were determined using an automated electrochemiluminescence immunometric assay on a Modular E170 Immunoanalyser (ECLIA, Roche Diagnostics, Mannheim, Germany). Lower limits of detection and CV's were $0.01 \mathrm{ng} / \mathrm{mL}$ and $2.6-5.6 \%$ for cTnT and $5 \mathrm{ng} / \mathrm{L}$ and 2.3-3.2\% for NT-proBNP. CK-MB (mass) was analysed in serum using an automated analyser IMx with the kit provided by the manufacturer (Abbott Diagnostics, IL, USA; detection limit $0.7 \mathrm{mg} / \mathrm{L}$ ). Concentration of ANP was determined using an immunoextraction (RIA) with a C-terminalspecific antiserum (Incstar, Stillwater, MN, USA; lower limit of detection $0.1 \mathrm{pmol} / \mathrm{L}, \mathrm{CV}: 6.8 \%-8.9 \%$ ) as previously described (Wondergem et al. 2001). In addition haemoglobin, electrolytes and creatinine concentrations were measured using routine methodology at the central laboratories for clinical chemistry of LUMC.

\section{Renal function}

Glomerular filtration rate was estimated using the MDRD formula: GFR $_{\text {estimate }}(\mathrm{mL} / \mathrm{min})=32,788 \times$ creatinine $(\mu \mathrm{mol} / \mathrm{L})^{-1.154} \times$ age $^{-0.203} \times$ constant $(1$ for males, 0.742 for females) (Levey et al. 1999).

\section{ECG recordings and analysis}

For each patient a 5-min ECG recording was made using the CardioPerfect device (Welch Allyn, Delft, The Netherlands). Heart rate (HR) and QT-interval were measured using the software supplied with the device. Care was taken to optimally assess the duration of the QT-interval as it has been reported that in $10-15 \%$ of cases ECG's fiducial points (e.g. P-onset, QRS-onset and end of T-wave) are not measured correctly by automated computer programs. Therefore the ECG recordings were additionally analysed after fiducial segment averaging (FSA) to obtain heart rate and QT-interval. This analysis was done using Intraval (Advanced Medical Systems, Maasdam, the Netherlands) (Ritsema van Eck. 2002).

For both analyses correction of the QT-interval for heart rate was done using Bazett's formula $(\mathrm{QTcB}=\mathrm{QT} / \sqrt{ }(\mathrm{RR}))$ and using the linear correction method according to Framingham $[\mathrm{QTcL}=\mathrm{QT}+0.154 \times(1-\mathrm{RR})]$. 


\section{Statistics}

Data from all patients were compared to the data obtained in the group of controls. The analysis of variance was conducted with factors group and gender and BMI and age as covariate, contrasts between the two groups were calculated along with $95 \%$ confidence intervals and least square mean estimates.

Data from the subpopulation of anthracyclin-naïve patients were analysed for changes occurring during the course (measurements at $t=0,4$ and 24) and whether there was a difference between courses. Two analyses were performed (Singal et al. 1997) analysis of variance with (within subject) factors time (0, 4 and 24), course and time by course using the data in original measurement units and (Shan et al. 1996) analysis of variance on change from baseline with baseline $(t=0)$ as covariate, with (within subject) factors time (4 and 24), course and time by course. Least square means were calculated for the different course/ time point combinations along with $95 \%$ confidence intervals.

In order to quantify the overall (average) time effect, the average change from baseline averaged over the four courses for the $t=4$ and 24 time point with $95 \%$ confidence interval was calculated within the ANOVA model.

All parameters, except for the ECG measurements, were $\log$ transformed prior to analysis to realise a normal distribution of the data and meet the requirements for ANOVA. In case of log transformation results were back-transformed resulting in geometric means and geometric mean ratios (for the contrasts and change from baseline). Geometric mean ratios were further translated into percentage change along with their $95 \%$ confidence intervals. Correlation analysis was performed on several biomarkers using regression analysis. All calculations were performed using SAS V9.1.2 (SAS Institute, Inc, Cary, NC, USA).

\section{Results}

\section{Biochemical markers}

Patients who completed chemotherapy had $277 \%(n=21$; 95\% CI: $86-661 \%, P<0.001)$ higher NT-proBNP levels in comparison to healthy volunteers $(n=14)$.

For the subpopulation of patients who were also followed during chemotherapy it was first investigated whether difference in volume loading resulted in different NT-proBNP responses. As this was not the case the data are given for the entire group. In these anthracyclin-naïve patients, the estimated increase in NT-proBNP was 269\% $(n=12 ; 167-409 \%, P<0.0001)$ at $24 \mathrm{~h}$ after the first DXR-course. Directly after completion of the DXR-infu- sion no increment was observed (Fig. 1). Similar two to threefold increases were found after the second, third and fourth course of DXR-therapy. NT-proBNP levels had returned to baseline before the start of each subsequent DXR course. CK-MB and ANP showed no differences between the groups, although there was a trend towards an increased ANP in the group of patients who already completed chemotherapy $(n=21)$ (Table 2). Renal function (estimated using the MDRD formula) was stable during the four courses and none of the subjects experienced clinical significant electrolyte abnormalities during the courses.

Anthracyclin-naïve patients had higher ANP and NTproBNP than the healthy controls $(n=14)$ by $140.8 \%$ $(n=12 ; 19.4-385.6 \%, P<0.02), 113.1 \% \quad(n=12 ; 8.0$ $320.1 \%, P<0.04)$, respectively.

cTnT concentrations were below the limit of detection in most cases and not further analysed for this reason.

In additional analyses the percentage change from baseline was calculated for all courses together (Table 3). Rises of $238 \%(n=12 ; 149-358 \%, P<0.0001), 44 \%(n=12 ; 4$ $101 \%, P<0.04), 26 \%(n=12 ; 4-52 \%, P<0.02)$ for NTproBNP, ANP and CK-MB were found at $24 \mathrm{~h}$.

There was no correlation between CK-MB on one hand and ANP and NT-proBNP.

\section{ECG}

No differences in HR and (corrected) QT-time could be observed when the patients who had completed the chemotherapy $(n=21)$ were compared with the healthy volunteers $(n=14)$ (Table 2). However there was a trend to a slightly prolonged QTc, corrected according to Bazett.

During each chemotherapy course (corrected) QT-time was prolonged immediately after completion of a course and remained so at $24 \mathrm{~h}$ after the DXR-course. As an exam-



Fig. 1 NT-proBNP, mean serum concentration (standard deviation) at baseline, 4 and $24 \mathrm{~h}$ in 12 patients who were sampled during each course of anthracyclin-containing chemotherapy 
Table 2 (Bio)markers and the differences between patients receiving at least three courses of anthracyclin-containing chemotherapy and healthy controls

\begin{tabular}{|c|c|c|c|c|c|c|}
\hline \multirow[t]{2}{*}{ Variable } & \multirow{2}{*}{$\begin{array}{l}\text { Controls } \\
(n=14)\end{array}$} & \multirow{2}{*}{$\begin{array}{l}\text { Patients } \\
(n=21)\end{array}$} & \multirow{2}{*}{$\begin{array}{l}\text { Patients versus } \\
\text { controls }\end{array}$} & \multicolumn{2}{|c|}{ 95\%-Confidence interval } & \multirow[t]{2}{*}{$P$ value } \\
\hline & & & & Lower & Upper & \\
\hline $\mathrm{ANP}(\mathrm{pmol} / \mathrm{L})$ & 11.3 & 20.7 & $82.9 \%$ & $-0.2 \%$ & $235.0 \%$ & 0.05 \\
\hline CK-MB mass (mg/L) & 1.1 & 0.9 & $-15.7 \%$ & $-48.1 \%$ & $37.0 \%$ & 0.48 \\
\hline NT-proBNP (ng/L) & 39.5 & 148.9 & $276.6 \%$ & $86.3 \%$ & $661.4 \%$ & $<0.001$ \\
\hline HR (bpm) & 67 & 71 & 4.20 & -1.95 & 10.36 & 0.17 \\
\hline QT (ms) & 400 & 400 & -0.31 & -18.1 & 17.47 & 0.97 \\
\hline QTc Bazett (ms) & 420 & 433 & 13.05 & -0.36 & 26.46 & 0.06 \\
\hline QTc linear (ms) & 414 & 422 & 8.22 & -2.68 & 19.13 & 0.13 \\
\hline
\end{tabular}

Average values of biochemical markers and ECG parameters and contrasts between anthracyclin-chemotherapy completers and controls, including 95\%-confidence intervals and $P$ values (for the biochemical markers contrasts are represented in percentage difference, for ECG parameters results are shown in absolute differences)

$A N P$ atrial natriuretic peptide, $C K-M B$ creatine kinase isoenzyme $\mathrm{MB}, N T$-proBNP N-terminal pro brain natriuretic peptide, $H R$ heartrate, $Q T$ QTinterval, QTc Bazett QT interval corrected according to Bazett, QTc linear QT interval corrected according to Framingham's linear correction method

Averages are estimated, as MIXED model analysis was used to correct for predose and missing values

Table 3 Summary table change from baseline (average of all four courses)

\begin{tabular}{|c|c|c|c|c|c|c|c|c|}
\hline & \multicolumn{4}{|l|}{$4 \mathrm{~h}$} & \multicolumn{4}{|l|}{$24 \mathrm{~h}$} \\
\hline & \multirow[t]{2}{*}{ Change } & \multicolumn{2}{|c|}{ 95\%-Confidence interval } & \multirow[t]{2}{*}{$P$ value } & \multirow[t]{2}{*}{ Change } & \multicolumn{2}{|c|}{ 95\%-Confidence interval } & \multirow[t]{2}{*}{$P$ value } \\
\hline & & Lower & Upper & & & Lower & Upper & \\
\hline $\mathrm{ANP}(\mathrm{pmol} / \mathrm{L})$ & $38.1 \%$ & $-0.3 \%$ & $91.3 \%$ & 0.05 & $44.3 \%$ & $3.6 \%$ & $101.2 \%$ & 0.03 \\
\hline CK-MB mass (mg/L) & $-2.2 \%$ & $-18.6 \%$ & $17.6 \%$ & 0.80 & $26.1 \%$ & $4.4 \%$ & $52.2 \%$ & 0.02 \\
\hline NT-proBNP (ng/L) & $18.0 \%$ & $-14.6 \%$ & $63.0 \%$ & 0.30 & $237.9 \%$ & $149.4 \%$ & $357.8 \%$ & $<0.001$ \\
\hline HR (bpm) & -0.29 & -3.37 & 2.79 & 0.85 & 1.46 & -1.67 & 4.60 & 0.34 \\
\hline QT (ms) & 10.95 & 3.91 & 17.98 & 0.004 & 10.96 & 3.77 & 18.15 & 0.005 \\
\hline QTc Bazett (ms) & 10.79 & 4.70 & 16.88 & 0.002 & 15.82 & 9.66 & 21.97 & $<0.001$ \\
\hline QTc linear (ms) & 10.04 & 5.39 & 14.69 & $<0.001$ & 13.13 & 8.40 & 17.85 & $<0.001$ \\
\hline
\end{tabular}

Average change (for all four courses) from baseline, 95\%-confidence intervals and $P$ values (for the biochemical markers results represent percentage change, for ECG parameters results are shown in absolute changes)

$A N P$ atrial natriuretic peptide, $C K-M B$ creatine kinase isoenzyme MB, $N T$-proBNP N-terminal pro brain natriuretic peptide, $H R$ heartrate, $Q T$ QTinterval, QTc Bazett QT interval corrected according to Bazett, QTc linear QT interval corrected according to Framingham's linear correction method

Averages are estimated, as MIXED model analysis was used to correct for predose and missing values

ple: during the first course QTc (using a linear correction method $)$ increased by $9.56 \mathrm{~ms} \quad(n=12 ; 3.85-15.27$, $P \leq 0.001)$ at completion and was increased by $11.48 \mathrm{~ms}$ $(n=12 ; 5.61-17.34, P \leq 0.0001)$ at $24 \mathrm{~h}$. Similar prolongations were observed during the subsequent courses. Heart rate did not change during the courses (Fig. 2). In the additional ECG-analyses using FSA, similar results were found.

\section{Discussion}

In the present study several markers that may be indicative for cardiac damage after DXR administration were investi- gated. The most important findings of this study are the observed change in NT-proBNP and prolonged QTc-intervals. This study indicates that at $24 \mathrm{~h}$ after each course of anthracyclin chemotherapy as well as after completion of a full chemotherapy regimen, significant increases in serum NT-proBNP are observed. Furthermore, the (corrected) QT-interval was prolonged with each course (course-tocourse prolongation).

NT-proBNP is cleaved from proBNP when it is converted into active BNP upon secretion. It is secreted equimolarly with BNP by the ventricle wall mainly in response to wall stretch and its secretion may be enhanced by catecholamine's, angiotensin II, endothelin and hypoxia (Hall 
A

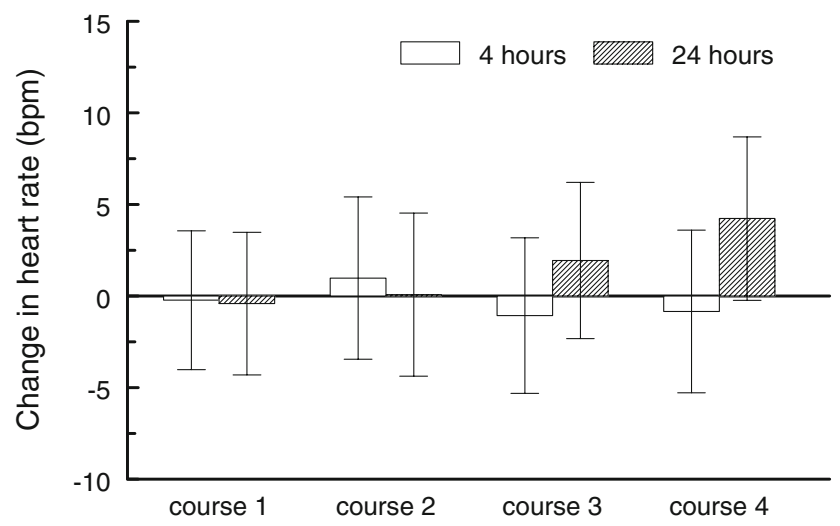

C

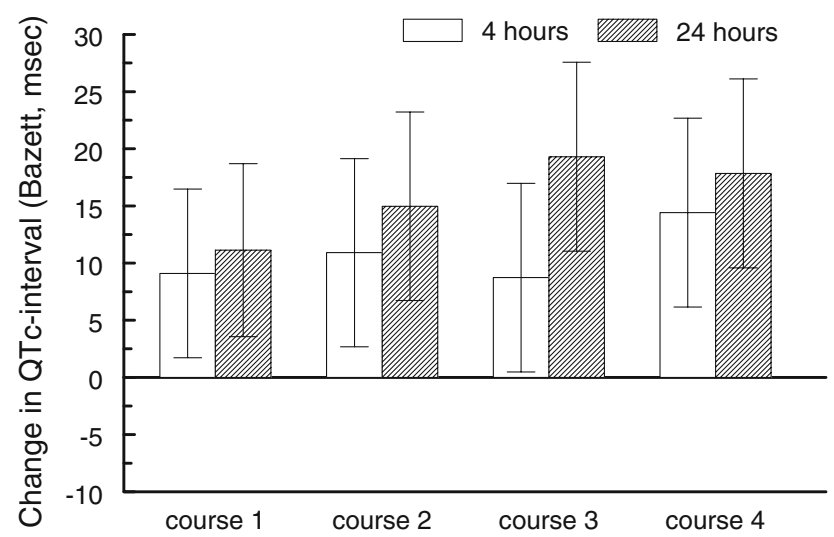

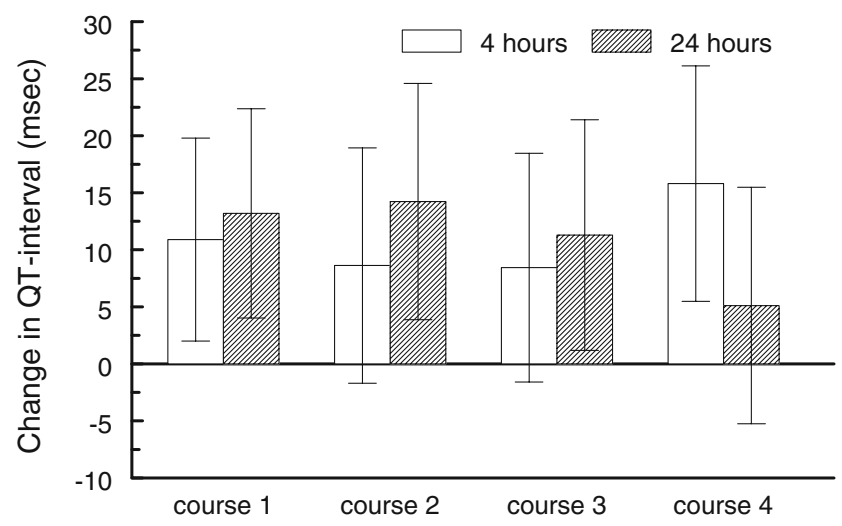

D

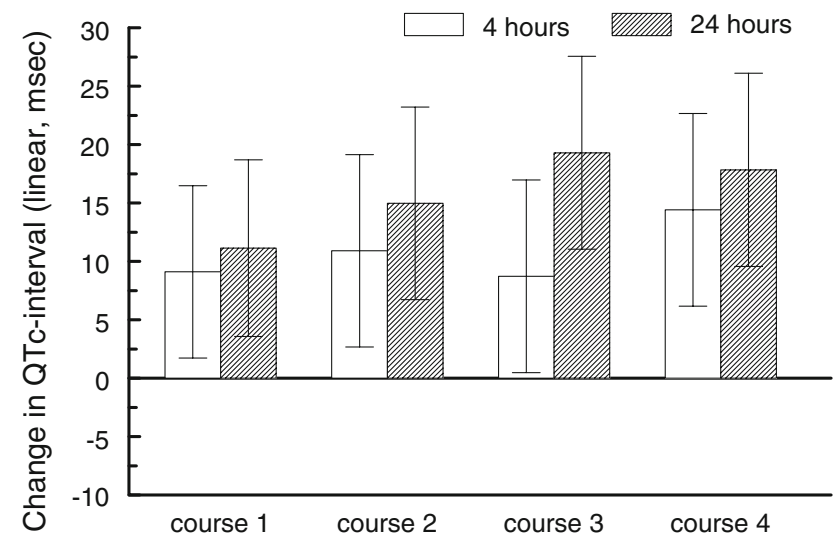

Fig. 2 Heart rate (beats per minute) and QT-intervals (milliseconds) change from baseline with 95\% CI error bars. a Heart rate, b QT-interval, c QTc-interval (Bazett), d QTc-interval (linear)

2005). NT-proBNP is a well established marker for CHF (Weber and Hamm 2006). Elevated levels of NT-proBNP (and BNP) are most notably associated with CHF, but also with other cardiac conditions (Silver et al. 2004). It is also known that concomitant kidney failure results in more pronounced elevations of NT-proBNP, especially in patients with an ejection fraction below 35\% (Luchner et al. 2005; Masson et al. 2006). As all included patients had a stable renal function during the study period, this could not explain the elevations of NT-proBNP in our study.

Elevation of concentrations of natriuretic peptides associated with previous exposure to anthracyclines has been described before, but this is mostly reported after completion of chemotherapy (Meinardi et al. 2001; Daugaard et al. 2005; Suzuki et al. 1998). An important additional finding of this study is that NT-proBNP is elevated relatively rapidly after exposure to anthracyclines. This effect was present for each individual subject, during each subsequent chemotherapy cycle. Therefore, we assume that DXR causes sufficient myocardial wall stress or neurohumoral responses on catecholamines or angiotensin to result in acutely elevated concentrations of NT-proBNP. This effect takes some time to develop as the NT-proBNP concentrations were unchanged immediately after the courses $(t=4 \mathrm{~h})$. However, elevated concentrations were invariably found in each patient at the $24 \mathrm{~h}$ time point. Although it cannot totally be excluded that the increases in NT-proBNP are (partly) caused by volume loading, this is highly unlikely as the infused volumes were low.

Additionally, the increase in NT-proBNP was transient as levels had returned to baseline at the subsequent course. This temporal relationship suggests that the initial effects of DXR on the heart are at least partly reversible during its first phases or compensated for by other mechanisms. This is plausible as animal studies indicated that the number of apoptotic cardiomyocytes after DXR show a biphasic pattern (Kumar et al. 2001). It is also in keeping with the notion that in clinical practice overt anthracyclin-induced cardiotoxicity develops after repeated exposure and failure of compensatory mechanisms (Minotti et al. 2004).

When all courses were taken together, an increase in ANP and CK-MB occurred after DXR. This effect could not be demonstrated for each individual patient-course combination, most likely because the increase in ANP var- 
ied between individuals and the study population was small. Furthermore cTnT level stayed below the detection limit in most cases. Both cTnT and CK-MB are widely used to assess myocardial injury in different clinical conditions (Malasky and Alpert 2002). There are conflicting data in the literature with regard to cTnT (Kilickap et al. 2005; Cardinale et al. 2004; Kismet et al. 2004; Fink et al. 1995; Lipshultz et al. 1997). A possible explanation for this discrepancy may be that many of the studies that failed to show an effect were small.

A surprising finding was that NT-proBNP and ANP were higher in the group of patients prior to anthracyclintherapy when compared to healthy volunteers. Missov et al. (1997) reported comparable results for cardiac Troponin I in patients with hematologic malignancies prior to anthracyclin-therapy. They suggested that patients with malignancies could experience some myocardial wall stress because of increased catecholamine release and an elevated sympathetic drive associated with anaemia and reflex tachycardia. However, our patients had normal haemoglobin concentrations, were normotensive, and had a normal heart rate, making this explanation for our population unlikely.

We also found significant prolongations of the QT-interval immediately after and at $24 \mathrm{~h}$ after each chemotherapy cycle. As it has been shown that in 10-15\% of cases ECG's fiducial points (e.g. P-onset, QRS-onset and end of T-wave) are not measured correctly by automated computer programs, the analysis of QT-intervals was performed using two methods which basically showed identical results (Ritsema van Eck 2002). The mechanism for the prolonged repolarisation of the heart after DXR-administration is unclear. Hypothetically, it may be a direct effect of DXR on cardiac repolarisation or the outcome of the loss of cardiomyocytes. The first explanation is supported by the observation that DXR is associated with acute arrhythmias and sudden cardiac death; however, there are no reports of DXR being a QT-prolonging drug. Although it is true that 5-HT3 receptor antagonists, which were used for the prevention of nausea, can prolong the QT-interval. However, QT-prolongations are present only very shortly after administration (up to $20 \mathrm{~min}$ ) and QT-intervals even decline thereafter (Charbit et al. 2005). Therefore 5-HT3 receptor antagonists cannot be responsible for the changes in QT-interval we observed at 4 and $24 \mathrm{~h}$ after the chemotherapy. The second explanation which links QT-interval prolongation to myocardial cell death may be more plausible. Whatever the cause of the QT-interval prolongation, it is certain that prolonged QT-intervals are linked to increased mortality and a variety of cardiac disorders, including heart failure (Brooksby et al. 1999).

Although our exploratory study may have identified promising markers to assess the course-to-course effects of DXR on the heart, it is important to also stress the limita- tions of this study. Obviously, the findings reported here should not be considered synonymous with anthracyclininduced cardiotoxicity, because no information is available to relate our findings to a measure of left ventricular function. However, it is unlikely that during the observation period of this study signs of ventricular dysfunction would have been detected as it is known that for the majority of the patients this develops over a longer period. Secondly, the patients included in this study received different dosing regimens and cytostatic drug combinations, which may have influenced the findings in this small cohort of patients. At present there is no indication that this occurred, but our group was probably too small to find such differences would they have been present.

In conclusion, the data indicate that NT-proBNP and the QT-interval are sensitive markers for the early detection of the course-to-course cardiac effects of DXR. Further research is needed to prospectively establish how the early changes in these markers relate to left ventricular dysfunction.

Open Access This article is distributed under the terms of the Creative Commons Attribution Noncommercial License which permits any noncommercial use, distribution, and reproduction in any medium, provided the original author(s) and source are credited.

\section{References}

Arola OJ, Saraste A, Pulkki K, Kallajoki M, Parvinen M, Voipio-Pulkki LM (2000) Acute doxorubicin cardiotoxicity involves cardiomyocyte apoptosis. Cancer Res 60:1789-1792

Bennink RJ, van den Hoff MJ, van Hemert FJ, de Bruin KM, Spijkerboer AL, Vanderheyden JL, Steinmetz N, Eck-Smit BL (2004) Annexin $\mathrm{V}$ imaging of acute doxorubicin cardiotoxicity (apoptosis) in rats. J Nucl Med 45:842-848

Brooksby P, Batin PD, Nolan J, Lindsay SJ, Andrews R, Mullen M, Baig W, Flapan AD, Prescott RJ, Neilson JM, Cowley AJ, Fox KA (1999) The relationship between QT intervals and mortality in ambulant patients with chronic heart failure. The United Kingdom heart failure evaluation and assessment of risk trial (UKHEART). Eur Heart J 20:1335-1341

Cardinale D, Sandri MT, Colombo A, Colombo N, Boeri M, Lamantia G, Civelli M, Peccatori F, Martinelli G, Fiorentini C, Cipolla CM (2004) Prognostic value of troponin I in cardiac risk stratification of cancer patients undergoing high-dose chemotherapy. Circulation 109:2749-2754

Charbit B, Albaladejo P, Funck-Brentano C, Legrand M, Samain E, Marty J (2005) Prolongation of QTc interval after postoperative nausea and vomiting treatment by droperidol or ondansetron. Anesthesiology 102:1094-1100

Daugaard G, Lassen U, Bie P, Pedersen EB, Jensen KT, Abildgaard U, Hesse B, Kjaer A (2005) Natriuretic peptides in the monitoring of anthracycline induced reduction in left ventricular ejection fraction. Eur J Heart Fail 7:87-93

Ewer MS, Benjamin RS (1996) Cardiotoxicity of chemotherapeutic drugs. In: Perry MC (ed). Baltimore, pp 649-663

Fink FM, Genser N, Fink C, Falk M, Mair J, Maurer-Dengg K, Hammerer I, Puschendorf B (1995) Cardiac troponin T and creatine kinase $\mathrm{MB}$ mass concentrations in children receiving anthracycline chemotherapy. Med Pediatr Oncol 25:185-189 
Hall C (2005) NT-ProBNP: the mechanism behind the marker. J Card Fail 11:S81-S83

Kilickap S, Barista I, Akgul E, Aytemir K, Aksoyek S, Aksoy S, Celik I, Kes S, Tekuzman G (2005) cTnT can be a useful marker for early detection of anthracycline cardiotoxicity. Ann Oncol 16:798804

Kismet E, Varan A, Ayabakan C, Alehan D, Portakal O, Buyukpamukcu M (2004) Serum troponin T levels and echocardiographic evaluation in children treated with doxorubicin. Pediatr Blood Cancer 42:220-224

Kumar D, Kirshenbaum LA, Li T, Danelisen I, Singal PK (2001) Apoptosis in adriamycin cardiomyopathy and its modulation by probucol. Antioxid Redox Signal 3:135-145

Launchbury AP, Habboubi N (1993) Epirubicin and doxorubicin: a comparison of their characteristics, therapeutic activity and toxicity. Cancer Treat Rev 19:197-228

Levey AS, Bosch JP, Lewis JB, Greene T, Rogers N, Roth D (1999) A more accurate method to estimate glomerular filtration rate from serum creatinine: a new prediction equation. Modification of diet in renal disease study group. Ann Intern Med 130:461-470

Lipshultz SE, Rifai N, Sallan SE, Lipsitz SR, Dalton V, Sacks DB, Ottlinger ME (1997) Predictive Value of Cardiac Troponin T in pediatric patients at risk for myocardial injury. Circulation 96:26412648

Luchner A, Hengstenberg C, Lowel H, Riegger GA, Schunkert H, Holmer S (2005) Effect of compensated renal dysfunction on approved heart failure markers: direct comparison of brain natriuretic peptide (BNP) and N-terminal pro-BNP. Hypertension 46:118-123

Malasky BR, Alpert JS (2002) Diagnosis of myocardial injury by biochemical markers: problems and promises. Cardiol Rev 10:306-317

Masson S, Latini R, Anand IS, Vago T, Angelici L, Barlera S, Missov ED, Clerico A, Tognoni G, Cohn JN (2006) Direct comparison of $\mathrm{B}$-type natriuretic peptide (BNP) and amino-terminal proBNP in a large population of patients with chronic and symptomatic heart failure: the Valsartan heart failure (Val-HeFT) data. Clin Chem 52:1528-1538

Meinardi MT, van Veldhuisen DJ, Gietema JA, Dolsma WV, Boomsma F, van den Berg MP, Volkers C, Haaksma J, de Vries EG, Sleijfer DT, van der Graaf WT (2001) Prospective evaluation of early cardiac damage induced by epirubicin-containing adjuvant chemotherapy and locoregional radiotherapy in breast cancer patients. J Clin Oncol 19:2746-2753

Minotti G, Menna P, Salvatorelli E, Cairo G, Gianni L (2004) Anthracyclines: molecular advances and pharmacologic developments in antitumor activity and cardiotoxicity. Pharmacol Rev 56:185229

Missov E, Calzolari C, Davy JM, Leclercq F, Rossi M, Pau B (1997) Cardiac troponin I in patients with hematologic malignancies. Coron Artery Dis 8:537-541

Ritsema van Eck HJ (2002) Fiducial segment averaging to improve cardiac time interval estimates. J Electrocardiol 35:89-93

Shan K, Lincoff AM, Young JB (1996) Anthracycline-induced cardiotoxicity. Ann Intern Med 125:47-58

Silver MA, Maisel A, Yancy CW, McCullough PA, Burnett JC Jr, Francis GS, Mehra MR, Peacock WF, Fonarow G, Gibler WB, Morrow DA, Hollander J (2004) BNP consensus panel 2004: a clinical approach for the diagnostic, prognostic, screening, treatment monitoring, and therapeutic roles of natriuretic peptides in cardiovascular diseases. Congest Heart Fail 10:1-30

Singal PK, Iliskovic N, Li T, Kumar D (1997) Adriamycin cardiomyopathy: pathophysiology and prevention. FASEB J 11:931-936

Suzuki T, Hayashi D, Yamazaki T, Mizuno T, Kanda Y, Komuro I, Kurabayashi M, Yamaoki K, Mitani K, Hirai H, Nagai R, Yazaki Y (1998) Elevated B-type natriuretic peptide levels after anthracycline administration. Am Heart J 136:362-363

Swain SM, Whaley FS, Ewer MS (2003) Congestive heart failure in patients treated with doxorubicin: a retrospective analysis of three trials. Cancer 97:2869-2879

Thorburn A, Frankel AE (2006) Apoptosis and anthracycline cardiotoxicity. Mol Cancer Ther 5:197-199

Von Hoff DD, Layard MW, Basa P, Davis HL Jr, Von Hoff AL, Rozencweig M, Muggia FM (1979) Risk factors for doxorubicin-induced congestive heart failure. Ann Intern Med 91:710-717

Weber M, Hamm C (2006) Role of B-type natriuretic peptide (BNP) and NT-proBNP in clinical routine. Heart 92:843-849

Wondergem J, Strootman EG, Frolich M, Leer JW, Noordijk EM (2001) Circulating atrial natriuretic peptide plasma levels as a marker for cardiac damage after radiotherapy. Radiother Oncol 58:295-301 